Cahiers $d u$ MONDE RUSSE

\section{Cahiers du monde russe}

Russie - Empire russe - Union soviétique et États indépendants

55/3-4 | 2014

Varia

\title{
Damaris Puñales-Alpizar, Escrito en cirílico, El ideal soviético en la cultura cubana posnoventa
}

Damaris Puñales-Alpizar, Écrit en cyrillique : l'idéal soviétique dans la culture cubaine après 1990

\section{Rafael Pedemonte}

\section{OpenEdition}

Journals

Édition électronique

URL : http://journals.openedition.org/monderusse/8150

DOI : $10.4000 /$ monderusse. 8150

ISSN : 1777-5388

Éditeur

Éditions de l'EHESS

Édition imprimée

Date de publication : 1 juillet 2014

Pagination : $523-527$

ISBN : 978-2-7132-2441-6

ISSN : $1252-6576$

Référence électronique

Rafael Pedemonte, «Damaris Puñales-Alpizar, Escrito en ciŕlico, El ideal soviético en la cultura cubana posnoventa », Cahiers du monde russe [En ligne], 55/3-4 | 2014, mis en ligne le 14 avril 2015, Consulté le 24 septembre 2020. URL : http://journals.openedition.org/monderusse/8150 ; DOI : https://doi.org/ $10.4000 /$ monderusse. 8150

Ce document a été généré automatiquement le 24 septembre 2020

(c) École des hautes études en sciences sociales 


\section{Damaris Puñales-Alpizar, Escrito en cirílico, El ideal soviético en la cultura cubana posnoventa}

Damaris Puñales-Alpizar, Écrit en cyrillique : l'idéal soviétique dans la culture cubaine après 1990

Rafael Pedemonte

\section{RÉFÉRENCE}

Damaris PUÑALES-ALPIZAR, Escrito en cirílico, El ideal soviético en la cultura cubana posnoventa, [Écrit en cyrillique : l'idéal soviétique dans la culture cubaine après 1990], Santiago : Cuarto Propio, 2013, 385 p.

1 L'Amérique latine n'a pas encore fait l'objet d'un grand intérêt de la part des spécialistes des relations internationales de l'Union soviétique. Le continent, considéré traditionnellement comme la zone du monde la moins importante aux yeux du Kremlin, a très rarement été incorporé dans les débats historiographiques sur les enjeux essentiels de la guerre froide ${ }^{1}$. Ces limites sont particulièrement évidentes en ce qui concerne l'analyse des rapports avec Moscou. Il est vrai qu'au cours de la guerre froide un certain nombre de chercheurs se sont penchés sur la question, mais la plupart demeuraient trop liés aux impératifs idéologiques de la période ; ils cherchaient avant tout à démontrer la supériorité du modèle politique représenté. Ces œuvres, rédigées surtout par des propagandistes soviétiques ou par des diplomates états-uniens, se sont, certes, multipliées considérablement mais en général elles n'ont fait que reproduire les thèses dominantes, à savoir le caractère marginal de l'Amérique latine dans la stratégie globale de l'URSS et la domination accablante imposée par la présence continentale de la superpuissance américaine. 
2 La chute du mur de Berlin a entraîné un certain renouveau en la matière, mais les publications restent encore insuffisantes. Ceci est d'autant plus frappant si l'on considère qu'en URSS de nombreuses archives relatives au domaine des affaires internationales ont été déclassifiées. On constate donc qu'au lieu d'inciter de nouvelles perspectives, la disparition de la puissance socialiste a découragé les recherches liées à sa présence sur le territoire latino-américain. Bien que l'on puisse dire sans trop d'hésitations que ces nations constituaient un enjeu secondaire pour l'URSS, cette assertion n'est pas applicable au cas tout à fait exceptionnel des rapports soviéto-cubains.

Pendant près de trente ans, l'île des Caraïbes est demeurée une source d'inquiétude permanente pour les leaders du Kremlin. En juillet 1972, La Havane devient membre du Conseil d'assistance économique mutuelle (CAEM), s'intégrant par ce biais dans le bloc communiste. La présence soviétique se fait désormais inépuisable: conserves d'aliments, vêtements, ouvrages spécialement traduits en espagnol, appareils électroménagers, voitures, etc., sont importés par milliers. Les contacts humains se multiplient aussi de façon exponentielle : près de quinze mille Cubains se forment en Union soviétique ${ }^{2}$ et des centaines de spécialistes russes débarquent dans le pays pour intégrer l'un des multiples programmes de coopération. Malgré l'intensité des relations et l'importance écrasante du référent soviétique pour la population, les experts semblent peu intéressés à se pencher sur les liens multiples établis entre les deux mondes. Au-delà des connexions politiques et des tensions diplomatiques qui ont caractérisé les années $1960^{3}$, - contexte qui a connu la crise dramatique des missiles, qui aurait pu avoir des conséquences apocalyptiques -, rares sont les aspects des rapports URSS-Cuba qui ont suscité un véritable intérêt académique.

Depuis quelques années cependant, une jeune génération de Cubains, issue surtout du domaine des études littéraires, tente de déchiffrer l'identité nationale à travers une grille de lecture qui insiste sur le rôle des références soviétiques ${ }^{4}$. C'est le cas de la critique littéraire et journaliste de l'université de La Havane, actuellement chercheuse de la Case Western Reserve (Ohio), Damaris Puñales-Alpízar. Elle consacre aujourd'hui des efforts considérables à analyser l'impact de la culture soviétique sur l'évolution identitaire de son pays après la disparition de l'URSS. Un volume qui vient d'être publié à Santiago du Chili (2013), rassemblant ses récentes contributions, mérite d'attirer notre attention.

Bien que l'objectif principal soit de s'interroger sur les particularités de la Cuba actuelle, l'auteur nous offre tout au long des près de 400 pages de l'ouvrage de multiples éléments pour comprendre l'intensité des relations culturelles soviéto-cubaines entre 1959 - année de l'arrivée au pouvoir de Fidel Castro et de ses hommes - et la fin de la guerre froide. Il s'agit ici d'une approche multidisciplinaire stimulante qui permet de dévoiler des facettes oubliées d'un sujet clé pour comprendre le caractère de la société cubaine : ses rapports culturels, humains et artistiques avec le " géant de l'Est ».

6 Damaris Puñales-Alpízar commence son livre par un intitulé dans un premier temps mystérieux mais qui s'éclaire par la suite: Confesiones postnacionales [Confessions postnationales]. Elle insiste sur le fait qu'à partir des années 1960, la présence culturelle soviétique se renforce de façon notoire (alors que celle des États-Unis s'affaiblit) et devient décisive pour la conformation de «l'homme cubain actuel». En tant qu'actrice $\mathrm{du}$ processus, elle se place au centre de ce qu'elle qualifie de «communauté 
sentimentale soviéto-cubaine ", concept clé autour duquel s'articule l'analyse. Ainsi - et en invoquant le professeur Harold Bloom pour qui toute critique littéraire est une forme d'autobiographie - elle remet en cause l'objectivité même de sa démarche.

Ayant défini ce cadre conceptuel, l'auteur évoque l'hypothèse principale qui enchaîne les différents chapitres de l'ouvrage : dès les premières années de la décennie de 1960, l'impact grandissant de l'URSS a permis de ressentir la culture de ce pays comme un élément indissociable de la vie quotidienne de l'île. De fait, la dimension colossale de cette présence humaine (de nombreux spécialistes, visiteurs, techniciens, professeurs universitaires se sont installés à Cuba), esthétique et matérielle engendre aujourd'hui une sorte de nostalgie à l'égard de cette période de collaboration accrue. Ceci est particulièrement évident en ce qui concerne les années 1980, quand la puissance socialiste envoyait de façon systématique toute une série de produits destinés à être consommés par les Cubains. Le contraste entre cette ère d'abondance et les difficultés déclenchées par suite de la chute de l'URSS, qui ont considérablement amoindri la qualité de vie, explique le surgissement d'un regard nostalgique porté sur la «phase soviétique ». Fusionné avec un certain degré d'admiration, cet attachement à la culture soviétique s'impose à présent dans la quotidienneté et ne peut se limiter aux simples convergences politiques.

8 Afin de démontrer ce postulat, Puñales-Alpízar fait appel à un ensemble de sources variées. Elle analyse notamment diverses manifestations artistiques contemporaines, liées d'une façon ou d'une autre, à l'URSS. Productions littéraires, œuvres d'art, films, chansons, récits de voyage, mémoires, etc., deviennent ainsi des vifs témoignages de la vigueur de l'ancienne présence soviétique. Mais elle ne s'intéresse pas seulement aux expressions actuelles. Nous pouvons aussi retracer tout au long du volume l'évolution et les vicissitudes des rapports culturels réciproques à partir de l'arrivée au pouvoir des révolutionnaires en janvier 1959. L'effort constant de mettre en perspective historique les connexions Cuba-URSS nous aide sans doute à mieux comprendre l'ancrage actuel de l'influence soviétique. L'interprétation des documents sélectionnés emprunte les méthodes heuristiques propres des historiens, mais se veut avant tout une critique littéraire et esthétique.

Dans son introduction, elle va chercher des sources explicatives dans le passé prérévolutionnaire, puis elle rend compte de l'ambiguïté qui a caractérisé les relations entre les deux États. En effet, on constate que dans l'évolution des rapports il n'y a pas toujours eu d'identification totale avec le modèle de Moscou. Bien au contraire, les productions artistiques témoignent des tensions ainsi que des tentatives d'explorer des voies esthétiques originales et même souvent hostiles aux doctrines soviétiques (le " réalisme socialiste » a été d'ailleurs maintes fois critiqué par les acteurs cubains). Suivant cette idée, elle établit quatre phases qui ont défini les contacts: les années 1960, marquées par le caractère indépendant et hétérogène de l'exploration artistique à Cuba; la décennie de 1970, quand on apprécie une convergence de plus en plus évidente avec le modèle culturel de l'URSS; les années 1980, témoins d'une phase d'ouverture et de détachement progressif à l'égard des fondements du "réalisme socialiste », et la période contemporaine - ultérieure à la disparition du bloc socialiste au cours de laquelle l'héritage de Moscou cesse d'avoir un contenu purement idéologique pour se transformer en objet privilégié d'expression artistique.

Le reste de l'ouvrage est composé de huit chapitres qui se focalisent sur des questions spécifiques tout en s'interrogeant sur le poids réel des «traces» de la culture 
soviétique dans la conformation de l'identité cubaine. Puñales-Alpízar évalue les formes locales de ce qu'elle qualifie de "réalisme socialiste tropical » (chap. 1) ainsi que la présence de thèmes associés à la réalité soviétique dans l'œuvre romanesque de l'ère révolutionnaire (chap. 2,4 et 5). Elle réserve également une place importante aux créations audiovisuelles, plus particulièrement à la coproduction cubano-soviétique Soy Cuba du fameux réalisateur Mihail Kalatozov (chap.7), à toute une série de documentaires récents, sans oublier l'impact que les dessins animés soviétiques ont eu sur l'imaginaire d'une génération de Cubains (chap. 6). Les liens humains sont aussi pris en compte pour montrer que les enfants des «couples mixtes» (surtout de femmes russes avec des hommes cubains) portent aujourd'hui une trace indélébile de cette double appartenance identitaire (chap. 3). Pour finir, l'auteur témoigne éloquemment de l'importation accélérée de productions matérielles issues du camp communiste (livres, voitures, électroménagers, boîtes de conserve alimentaire, matériaux de construction, etc.) et surtout du choc provoqué par la disparition soudaine de ces produits après la guerre froide (chap. 8 ).

11 Après l'analyse des cas, l'auteur conclut en réaffirmant l'existence d'une « communauté sentimentale soviéto-cubaine", indiquant tout de même que cette particularité n'implique guère une forme de domination ou d'assimilation passive de la culture de l'Est. Bien au contraire, elle insiste sur la capacité constante de se réapproprier des manifestations allogènes. Dans ce sens, le référent soviétique a été maintes fois remémoré mais pas pour autant idéalisé par les habitants de l'île. Il s'agit là d'une idée séduisante qui stimule certainement un questionnement renouvelé des rapports Cuba-URSS et qui nous incite à porter un regard attentif sur les effets locaux de la "pénétration culturelle » des puissances. Mais malgré son intérêt incontestable, nous aurions voulu voir davantage d'indices concrets qui nous permettent de mieux corroborer l'hypothèse. L'étude présente de nombreuses affirmations tout à fait suggestives mais souvent dépourvues d'un corpus d'antécédents solides. Réaliser des recherches dans des centres d'archives - tant du côté russe que cubain - serait sans doute une démarche souhaitable pour multiplier les données à l'égard du phénomène. L'ouvrage est un premier pas sérieux et tout à fait stimulant, dont Damaris Puñales-Alpízar détient tout le mérite. Cependant, les preuves demeurent, pour l'instant, insuffisantes. Elle nous ouvre un champ intéressant, appelé à être approfondi et à devenir un domaine d'étude privilégié.

\section{NOTES}

1. Gilbert Joseph, « What We Know and Should Know : Bringing Latin America More Meaningfully into Cold War Studies », in Joseph Gilbert, Daniela Spencer, eds., In From the Cold: Latin America's New Encounter with the Cold War, Durham : Duke University Press, 2008, p. 9-10.

2. Alexander Moiseev, Olga Egorova, Los rusos en Cuba. Crónicas históricas : juicios y testimonios, La Habana : Editora Abril, 2010, p. 119.

3. Voir par exemple James Blight, Philip Brenner, Sad \& Luminous Days : Cuba's Struggle with the Superpowers after the Missile Crisis, Lanham : Rowman \& Littlefield, 2002 ; Aleksandr Fursenko, 
Timothy Naftali, “One Hell of Gamble” : Khrushchev, Castro, and Kennedy, 1958-1964, New York : Norton, 1997 ; Leila Latrèche, Cuba et l'URSS : 30 ans d'une relation improbable, P. : L'Harmattan, 2011.

4. Voir Jacqueline Loss, Dreaming in Russian : The Cuban Soviet Imaginary, Austin : University of Texas Press, 2013, ainsi que le numéro spécial de la revue Gaceta de Cuba, (1) 2001.

\section{AUTEURS}

\section{RAFAEL PEDEMONTE}

Université Paris 1 Panthéon-Sorbonne (CRHS) - université catholique du Chili 